\title{
COVID-19 IN PEDIATRIC CANCER PATIENTS IN A RESOURCE-LIMITED SETTING: NATIONAL DATA FROM PERU
}

Jacqueline Montoya $^{1}$, Cecilia Ugaz ${ }^{1,2}$, Sandra Alarcon ${ }^{1}$, ESSY MARADIEGUE ${ }^{1}$, Juan Garcia $^{1}$, Rosdali Diaz ${ }^{1}$, Arturo Zapata ${ }^{1}$, Sharon Chavez $^{1}$, Roxana Morales ${ }^{1}$, Katy Ordoñez ${ }^{1}$, Eddy Hernandez ${ }^{1}$, Romulo Reaño ${ }^{1}$, María Pía Vargas ${ }^{1}$, Cynthia Gutierrez ${ }^{1}$, Katherine Sanchez ${ }^{1}$, Cinthya Valdiviezo ${ }^{1}$, Ninoska Rojas ${ }^{3}$, Carla Moore ${ }^{4}$, Esmeralda Leon ${ }^{5}$, Ivan $\mathrm{Maza}^{3}$, and Liliana Vasquez ${ }^{6}$

${ }^{1}$ Instituto Nacional de Enfermedades Neoplasicas

${ }^{2}$ Pediatric Blood \& Cancer Open Research

${ }^{3}$ Hospital Nacional Edgardo Rebagliati Martins

${ }^{4}$ Instituto Nacional de Salud del Nino San Borja

${ }^{5}$ Level IV Hospital Guillermo Almenara Irigoyen

${ }^{6}$ PAHO Perú

July 9, 2020

\section{Hosted file}

LETTER TO THE EDITOR COVID EN CHILDREN WITH CANCER PERU.docx available at https://authorea. com/users/341213/articles/468298-covid-19-in-pediatric-cancer-patients-in-a-resourcelimited-setting-national-data-from-peru

\section{Hosted file}

Table 1.docx available at https://authorea.com/users/341213/articles/468298-covid-19-inpediatric-cancer-patients-in-a-resource-limited-setting-national-data-from-peru 